\title{
Flight Formation of UAVs in Presence of Moving Obstacles Using Fast-Dynamic Mixed Integer Linear Programming
}

\author{
Mohammadreza Radmanesh *, Manish Kumar ${ }^{\dagger}$
}

\begin{abstract}
This paper proposes the implementation of fastdynamic Mixed Integer Linear Programming (MILP) and Path Smoother for efficient path planning of Unmanned Aerial Vehicles (UAVs) in various flight formations. The UAVs taking part in a cooperative flight are assumed to be equipped with Automatic Dependent Surveillance Broadcast (ADS-B) which enables sharing the flight information with neighboring aircraft. The design and implementation of flights for various formations have been carried out in a generic manner such that multiple UAVs with arbitrarily geographically located base stations can take part in collision-free formation flight. The paper formulates the problem of path of planning in the framework of a novel fast-dynamic MILP and proposes a cost function that minimizes time and energy consumption. The paper presents elaborate construction of constraint equations to enforce the formation to visit pre-defined way-points and avoid the collisions with any intruder aircraft. The performance of the proposed algorithm has been verified and compared with respect to the standard MILP method via a number of simulations carried out using different scenarios featuring multiple UAVs flying in various formations.
\end{abstract}

Key Words: UAV; NAS; MILP; ADS-B; Trajectory Planning; Flight Formation.

${ }^{*}$ Corresponding Author, Graduate Research Assistant, Cooperative Distributed Systems Lab, Department of Mechanical and Materials Engineering, University of Cincinnati, Cincinnati, Ohio 45221, USA, Email: radmanma@mail.uc.edu

${ }^{\dagger}$ Associate Professor, Cooperative Distributed Systems Lab, Department of Mechanical and Materials Engineering, University of Cincinnati, Cincinnati, Ohio 45221, USA, Email: manish.kumar@uc.edu

\section{Introduction}

Unmanned Air Vehicles (UAVs) have traditionally been used in military operations for a number of years. Recently, UAVs have generated a lot of interest due to their potential application in civilian domains such as emergency management, law enforcement, precision agriculture, package delivery, and imaging/surveillance $[45,31,37,29]$. However, before the use of UAVs becomes a reality in civilian domains, the challenges emanating from integration of UAVs in the National Airspace System (NAS) are extremely critical to be solved. An important among these challenges is the ability for a UAV to not only plan its own path for fulfilling a mission but also to replan or adjust its trajectory in order to avoid collision with other aircraft. Furthermore, the increase in the number of aircraft has been dramatic over the last 50 years. This increase in manned aircraft along with incorporation of unmanned fleet in future will pose severe challenges to the current Air Traffic Control (ATC). Hence, the Radio Technical Commission for Aviation (RTCA) and also Federal Aviation Administration (FAA) have been charged with a responsibility to implement a seamless change from ATC to Air Traffic Management (ATM) by $2020[39,34,32]$ that incorporates mechanisms to plan/replan the paths of UAVs to avoid collisions with other aircraft.

There are various methods for calculating escape trajectories that have been proposed for collision avoidance including classical control [4], Fuzzy Logic [28], E-Field maneuver planning [27, 40], game theory [47] and their application in NC Machines path planning [11, 49], automotive trajectory planning [2], and air traffic management [13]. 
Group cooperative behavior implies that the members share a common goal and act according to the common objective of the group. Effective cooperation often requires that each individual of the group coordinates its actions [33]. Using multiple UAVs for the different applications has attracted many researchers. Apart from the fact that multiple UAVs provide ability to perform complex and heterogeneous tasks, one of the advantages of cooperative flight performances is also fuel saving [8, 12]. Path planning of such systems offers many challenging problems from both theoretical and practical points of view. Formation flight is referred to a particular problem of management of a group of UAVs flying in tight cooperation within a defined volume [23], and often with a pre-defined shape. Although studies on active path planning of a UAV have been considered many times (e.g., see $[17,50,51]$ ), cooperative path planning approaches for UAVs have only recently begun to appear. The problem of formation flight is widely studied in literature. Considering only the flight control, classical leader-wingman configuration is investigated via proportional-integral control [9] or non-linear control [46]. A reactive behaviorbased controller is discussed in [5]. Proposed solution for trajectory optimization of large formations using centralized or distributed algorithms is discussed respectively in $[25,38]$, taking into account some constraints on the shape of the formation. Reconfiguration in the formations is introduced in Reference [53] by proposing a scheme where trajectories are computed off-line for switching between a limited number of formation configurations. In $[42,1,19,18]$, by implementation of mixed-integer linear programming (MILP), tightly-coupled task assignment problems with timing constraints is solved for a group of UAVs.

This paper focuses on developing a method for a team of UAVs, in this case quad-copters, to navigate through an environment filled with static and dynamic obstacles while in formation. The proposed method formulates the path planning problem in the framework of MILP, the solution of which provides the waypoints for each UAVs. The main contribution of the paper is proposing a fast-dynamic approach to the MILP using a hybrid branch and bound method for obtaining exact solution of the MILP over rational numbers. Since the aim is to exactly and efficiently solve MILP with application to UAV trajectory planning, a version of branch-and-bound is proposed that attempts to combine the advantages of the pure rational and safe-Floating Point (FP) approaches, and compensates for their individual weaknesses. The solution is to work with two different and hybrid branch-and-bound processes. The aim of the main process is to implement the rational approach. The other part of the process is the slave process where the faster FP approach is applied. Furthermore, a concept of using dynamic finite horizon is implemented in the paper that solves the MILP is a local spatial region that keeps updating as the UAV continues on its path.

A cost function is proposed that includes two components: i) total time to minimize the time of flight; and ii) control inputs to minimize energy consumption. The paper then implements a path smoothing strategy to adapt the generated path to the dynamics of the UAV. The paper considers two scenarios of flight formations in order to simulate flight performances. In the first scenario, the UAVs break the formation in presence of obstacles and try to get to their goal path while minimizing the cost function. In the second scenario, the constraint of the fixed formation is applied on UAVs for the whole duration of flight so that UAVs navigate from initial point to final point while maintaining the formation and minimizing the cost function. At the end of each flight formation the time of computation for each scenario has been compared.

\section{Problem Formulation and General Approach}

In this paper, $c \in[1, \ldots, C]$ represents the index for individual UAVs cooperating in the formation. Let $t_{=}[1,2, \ldots, T]$, be the time step and $T$ is the time taken by the entire team of UAVs to reach the local goal position. $w_{c} \in\left[1, \ldots, W_{c}\right]$ is the index for the waypoint needed to be visited by a UAV $c$. $T_{\text {total }}$ is the total time of flight from the starting point to the global 
goal position. Let us define $b_{\text {final }}$ to be the binary variable for visiting the goal point. We choose our cost function as follows:

$$
\begin{array}{r}
\forall t \in[1, \ldots, T] \in T_{\text {total }}, \forall c \in[1, \ldots, C] \\
J_{\text {min,time }}=\sum_{c=1}^{C} \sum_{t=1}^{T_{\text {total }}}\left[b_{\text {final }} T+\varepsilon_{1}\left(\left|f_{x, t}\right|\right)\right. \\
\left.+\varepsilon_{2}\left(\left|f_{y, t}\right|\right)+\varepsilon_{3}\left(\left|f_{z, t}\right|\right)\right]
\end{array}
$$

$\varepsilon_{1}, \varepsilon_{2}$ and $\varepsilon_{3}$ are the penalties for the fuel consumption and small enough to ensure that the penalty never exceeds the value of each time step. $f_{x, t}, f_{y, t}$ and $f_{z, t}$ are also the components of the forces exerted on the quad-copters body due to propellers' rotation at the time step $t$. The objective of this cost function is to minimize combination of the fuel consumption and time. $b_{\text {final }}$ is active (equal to 1 ) only when each UAV reaches its final destination otherwise it is inactive (equal to 0 ). In other words, the UAVs reaching the final destination can be represented by the constraint equation:

$$
\sum_{i=1}^{T} b_{\text {final }}=1
$$

In our approach, the MILP is used to formulate the path planning problem for a single UAV or a group of UAVs navigating from the initial position to the destination. MILP strategies are enhanced by powerful solvers which have now been developed and freely available. Powerful packages like CPLEX or Gurobi would solve MILPs efficiently for reasonably sized problems. One of the disadvantages of MILP is that the strategy is NP-hard, and thus computations would grow considerably if the dimensionality of the decision variables needed to model the problem increases. In this study, the optimization problem and the constraints are written in the YALMIP language [26] and the solver will be Gurobi optimizer.

\subsection{Detecting an Intrusion or Colli- sion}

Since the direction of the neighboring aircraft, called Intruder Aircraft (IAs), might change with time, detection of intrusion needs to be in an online/real-time fashion. This means the collision point, the threat area and the ADS-B data should be updated or predicted in an online and dynamic way. The threat area, as shown in Figure 1, is defined as the area around the UAV which when breached by any aircraft, indicates that aircraft as an intruder aircraft. Basically detection of a collision begins with the received ADS-B data, that includes position and velocity of IA, from the threat area. The data obtained from ADS-B is used to estimate the states, i.e., position, velocity, and acceleration of the IA via a Kalman filter. Using a Kalman filter to estimate the states helps in reducing the effect of noise in the ADS-B data. The 3-D position of IA at time $t$ is represented as $\left(x_{I A, t}, y_{I A, t}, z_{I A, t}\right)$ and the position of UAV $c$ at time step $t$ is represented by $\left(x_{c, t}, y_{c, t}, z_{c, t}\right)$ along the predefined path. The collision is defined as:

$$
\begin{array}{r}
\forall c \in[1, \ldots, C], \\
\left(x_{I A, t}, y_{I A, t}, z_{I A, t}\right) \in\left(x_{c, t}, y_{c, t}, z_{c, t}\right)_{\text {predefinedpath }}
\end{array}
$$

\subsection{Defining the Finite Horizon}

The finite horizon is used for limiting the area around the UAV, large enough to allow the UAV to maneuver in order to avoid a collision and small enough so that the computational time does not increase enormously. The appropriate amount of time needed for avoiding a collision depends on the velocity of the UAV, weather conditions, the expected position of the collision and the velocity of the IA. Furthermore, IA area represents the communication range where ADS-B data can be received from the other aircraft. Figure 1 shows a schematic diagram of the IA area and Threat Area.

A local goal is defined as the intersection of the predefined path and the finite horizon. Two cases are considered here and finite horizons are defined differently for these cases. The first case is when the IA's velocity vector is not aligned as the velocity vector of the UAV $c$ and the second case is when the IA's velcoity is aligned with that of the UAV. 


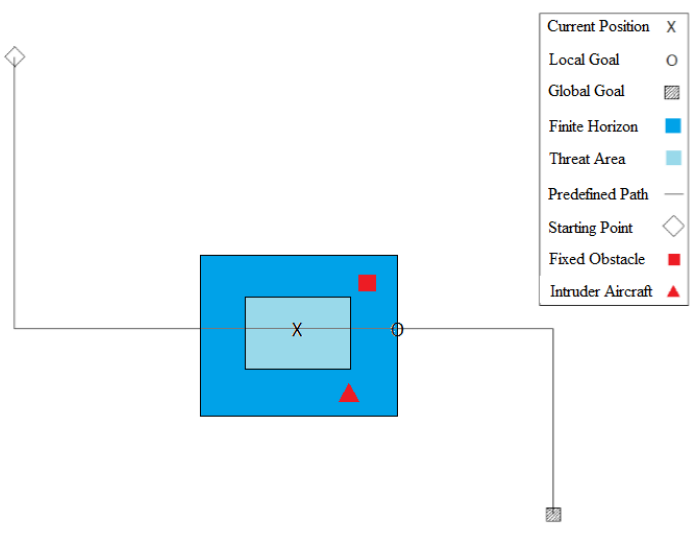

Figure 1: The schematic diagram of threat area and IA area

\subsubsection{IAs Approaches the Formation not on the Same Line of Flight}

In this case, for the UAV, flies in the finite horizon from $t_{\text {collision }}$ seconds before the first anticipated collision. The calculation of $t_{\text {collision }}$ is based on the assumption that the UAV flies with the maximum velocity of $v_{\max , \text { forward }}$ and $v_{\max , z}$. The finite horizon ends at the time at which the UAV reaches the local goal. $t_{\text {collision }}$ can be found from:

$$
\begin{array}{r}
\forall t \in\left[t_{1}, t_{1}+1, \ldots, t_{G}\right], \\
0 \leq t_{1} \leq t_{G} \leq T_{\text {total }} \\
\left(x_{t_{\text {collision }}}, y_{\left.t_{\text {collision }}, z_{t_{\text {collision }}}\right)=\left(x_{c, t}, y_{c, t}, z_{c, t}\right)}^{1}\right] \\
t_{\text {collision }}=\left(\left[\frac{1}{\left(v_{\text {max }, \text { forward }}^{2}+v_{\text {max }, z}^{2}\right)}\right]\right. \\
{\left[\left(x_{\text {collision }}-x_{t_{1}}\right)^{2}+\left(y_{\text {collision }}-y_{t_{1}}\right)^{2}\right.} \\
\left.\left.\left.+\left(z_{\text {collision }}-z_{t_{1}}\right)^{2}\right)\right]\right)^{\frac{1}{2}}
\end{array}
$$

The suggested finite horizon is modeled as a cube of length $L_{2}$ given by:

$$
\begin{gathered}
\forall o \in[1, \ldots, O], \\
L_{2}=Q_{2} * V_{r e l, o, t}
\end{gathered}
$$

If there is a possibility of multiple IAs from the front, only the highest $V_{r e l, o, t}$ needs to be considered. $Q_{2}$ is again an experimental factor needed to be considered for the computation. $\left(x_{G}, y_{G}, z_{G}\right)$ is the intersection of the predefined path and the defined cube. 
In the second case, the IA approaches the UAV from behind. This condition only applies when the $v_{t, I A}>v_{t}$. The proposed finite horizon for this case is a cube centered at the current position of the UAV with length $L_{3}$ given by:

$$
\begin{array}{r}
V_{r e l, o, t}=v_{o, t}-v_{c, t} \\
L_{3}=Q_{3} \times V_{r e l, o, t}
\end{array}
$$

We also calculate $L_{4}$ as:

$$
L_{4}=Q_{4} \times V_{\max , o, \text { total }}
$$

And $Q_{2}, Q_{3}, Q_{4}$ are all greater than one. In order to avoid collisions, the strategy is to make the biggest possible finite horizon which is done in the following way:

$$
L_{\text {total }}=\max \left[L_{1}, L_{2}, L_{3}, L_{4}\right]
$$

In this finite horizon area, the start point and the final goal are located at the predefined path and the UAVs are trying to avoid collision in this part.

\subsection{UAV Dynamics}

The mathematical statements of translational movements about the Center of Gravity (CG) written for the body $\{b\}$ frame of the UAV is described in Reference [20]:

$$
\begin{aligned}
m\left(\dot{v}^{b}+w_{g / n}^{b} \times v^{b}\right)+D v^{b} & =m R^{T} g^{n}+\tau^{b},(11) \\
& +\tau_{\text {wind }}^{b}+\tau_{\text {other }}^{b}
\end{aligned}
$$

where $m$ is the mass of the $\mathrm{UAV}, D$ is the matrix of positive definite damping, $g^{n}$ is gravity vector in North East Down (NED) frame, $v^{b}$ is the velocity in body frame, $\tau^{b}$ is the control inputs of the aircraft and $\tau_{\text {wind }}^{b}$ and $\tau_{\text {other }}^{b}$ are the forces of the aircraft body caused by wind and other disturbances respectively. Also, $R$ is the rotation matrix in body frame to the NED frame. By using the skew-symmetric matrix:

$$
m\left(\dot{v}^{b}+S\left(w_{g / n}^{b}\right) v^{b}\right)+D v^{b}=m R^{T} g^{n}+\tau
$$

The derivative of the velocity component in the NED frame can be cast as:

$$
\dot{v}^{n}=R\left(\dot{v}^{b}+w^{b} \times v^{b}\right)
$$

Multiplying equation 12 by $R$ and substitution in equation 11 results in:

$$
\dot{v}^{n}=-\frac{1}{m} \delta \nu^{n}+g^{n}+\frac{1}{m} \tau_{\text {wind }}^{n}+\frac{1}{m} \tau_{\text {other }}^{n}
$$

where:

$$
\delta:=R D R^{T}
$$

The problem considered in this paper is with $\tau_{\text {wind }}^{n}=$ 0 and $\tau_{\text {other }}^{n}=0$. Since the solution is considered only for a 2-D problem, $g$ can be eliminated from the equations. The result in the NED frame is:

$$
\left[\begin{array}{c}
\dot{v}_{x} \\
\dot{v}_{y}
\end{array}\right]=\frac{1}{m}\left(-\delta^{T}\left[\begin{array}{l}
v_{x} \\
v_{y}
\end{array}\right]+\left[\begin{array}{c}
\tau_{x} \\
\tau_{y}
\end{array}\right]\right)
$$

The following Lyapunov function is used to analyze the stability properties:

$$
V(t)=\frac{1}{2} v^{t} v>0, \forall v \neq 0
$$

where $V(T)$ is positive definite and is zero only when $v=\left[\begin{array}{lll}v_{x} & v_{y} & v_{z}\end{array}\right]^{t}=0$. By this means the derivative of Lyapunov function for the unforced system $(\tau=0)$ is gained from the equation 18 [24].

$$
\dot{V}(t)=-v^{t} \frac{1}{m} \delta v
$$

Since $V \dot{(t)}<0$ the case is Globally Exponentially Stable (GES) [21]. By removing the damping from the equation (i.e., $\delta=0$ ) and considering forced system (i.e. $\tau \neq 0$ ), the Lyapunov derivative can be written as:

$$
\dot{V}(t)=\frac{1}{m} v^{t} \tau
$$

Where $\tau=\left[\begin{array}{lll}\tau_{x} & \tau_{y} & \tau_{z}\end{array}\right]$. In this case, stability will depend on the chosen control force. GES property is achieved, for example, by requiring that $\tau=-v$. 
The result is:

$$
\begin{aligned}
{\left[\begin{array}{c}
\dot{x} \\
\dot{y} \\
\dot{z} \\
\ddot{x} \\
\ddot{y} \\
\ddot{z}
\end{array}\right]=} & {\left[\begin{array}{llllll}
0 & 0 & 0 & 1 & 0 & 0 \\
0 & 0 & 0 & 0 & 1 & 0 \\
0 & 0 & 0 & 0 & 0 & 1 \\
0 & 0 & 0 & 0 & 0 & 0 \\
0 & 0 & 0 & 0 & 0 & 0 \\
0 & 0 & 0 & 0 & 0 & 0
\end{array}\right]\left[\begin{array}{c}
x \\
y \\
z \\
\dot{x} \\
\dot{y} \\
\dot{z}
\end{array}\right] } \\
+ & {\left[\begin{array}{llll}
0 & 0 & 0 \\
0 & 0 & 0 \\
0 & 0 & 0 \\
\frac{1}{m} & 0 & 0 \\
0 & \frac{1}{m} & 0 \\
0 & 0 & \frac{1}{m}
\end{array}\right]\left[\begin{array}{l}
f_{x} \\
f_{y} \\
f_{z}
\end{array}\right] }
\end{aligned}
$$

Equation 20 can be written as:

$$
\dot{s}=A s+B u
$$

where:

$$
s:=\left[\begin{array}{llllll}
x & y & z & \dot{x} & \dot{y} & \dot{z}
\end{array}\right]^{T}
$$

The result has full compatibility with Newtons second law and the discrete form of Equation 21 can be written as $[10,7]$ :

$$
\begin{array}{r}
\forall t \in[0, \ldots, T] \\
s_{t+1}=A_{d} s_{t}+B_{d} u_{t} \\
s_{0}=s_{I}
\end{array}
$$

where $T$ represents the time taken by UAVs to go through the finite horizon $A_{d}$ and $B_{d}$ are the discrete system matrix and discrete input matrix respectively. Furthermore, $s_{I}=\left[\begin{array}{llllll}x_{0} & y_{0} & z_{0} & v_{x, 0} & v_{y, 0} & v_{z, 0}\end{array}\right]^{T}$ is the initial state and $u=\left[\begin{array}{lll}f_{x, t} & f_{y, t} & f_{z, t}\end{array}\right]^{T}$. The spatial region of UAVs motion has been considered as in the earlier section and the constraints that should be satisfied in 3-Dimensions are defined as a cubic region as follows:

$$
\begin{array}{r}
\forall c \in[0, \ldots, C], \forall t \in[0, \ldots, T] \\
x_{\min } \leq x_{t, c} \leq x_{\max } \\
y_{\min } \leq y_{t, c} \leq y_{\max } \\
z_{\min } \leq z_{t, c} \leq z_{\max }
\end{array}
$$

For the constraints on the velocity, components of velocity in $x, y$, and $z$ directions have been considered. According to the nature of flight, UAVs usually move slower in $z$ direction as compared to the $x$ and $y$ directions. Furthermore, the result in NED frame neglects the UAV orientation. The constraint on velocity can be expressed as:

$$
\begin{gathered}
v_{\text {min,forward }}^{2} \leq v_{x, t, c}^{2}+v_{y, t, c}^{2} \leq v_{\text {max }, \text { forward }}^{2} \\
v_{z, \min } \leq v_{z, t, c} \leq v_{z, \max }
\end{gathered}
$$

where $v_{x, t, c}, v_{y, t, c}$ and $v_{z, t, c}$ are the velocity components of UAV $c$ at time $t$. For the non-linear constraints, according to the [7] the equation 27 is approximated using a constant number of $H$ by:

$$
\begin{aligned}
& \forall t \in[0, \ldots, T-1], \forall h \in[1, \ldots, H], \forall c \in[0, \ldots, C] \\
& v_{\text {min }, \text { forward }} \leq v_{x, t, c} \sin \left(\frac{2 \pi h}{H}\right)+v_{y, t, c} \cos \left(\frac{2 \pi h}{H}\right) ; \\
& v_{x, t, c} \sin \left(\frac{2 \pi h}{H}\right)+v_{y, t, c} \cos \left(\frac{2 \pi h}{H}\right) \leq v_{\text {max }, \text { forward }}
\end{aligned}
$$

Same conditions apply for the forces exerted on the UAVs. Furthermore, due to engine limitation, constraints on the maximum force need to be considered which includes i) The maximum centripetal force due to bank turn and ii) The maximum forward force from the UAV engine.The constraints on force can be expressed as:

$$
f_{x, t, c}^{2}+f_{y, t, c}^{2} \leq f_{\max }^{2}
$$

It is noted that $f_{z, \max }$ should be more than the weight of the airplane.

$$
0 \leq f_{z, t, c} \leq f_{z, \max }
$$

Using the same method, to arrange the linear form of this constraint is formed as in Equation 32 [36].

$$
\begin{gathered}
\forall t \in[0, \ldots, T-1], \forall h \in[1, \ldots, H], \forall c \in[0, \ldots, C] \\
-f_{\max } \leq f_{x, t, c} \sin \left(\frac{2 \pi h}{H}\right)+f_{y, t, c} \cos \left(\frac{2 \pi h}{H}\right) \leq f_{\max }
\end{gathered}
$$

By increasing the $H$, the shape of the approximation gets closer to a circle which is close to the true constraint. 


\subsection{Defining the Constraints for Maintaining Separation with Intruder Aircraft}

As described by [48], the UAVs can fly in a finite horizon and can face fixed obstacles or moving obstacles which are called as the intruder aircraft (IAs). In this study, the boundaries of the obstacles, have been increased for the amount of $2 * \sigma$ in each direction for safety factor.

For implementation in MILP, the constraints are obtained by defining a large positive number $M_{b i g}$ which is much bigger than difference between any pair of waypoint positions and UAV positions. Constraints are defined by $[10,22]$ :

$$
\begin{array}{r}
\forall t \in[0, \ldots, T], \forall o \in[1, \ldots, O], \forall c \in[1, \ldots, C], \\
x_{t, c} \leq x_{\min , o}+M_{b i g} t_{t, o, 1} \\
-x_{t, c} \leq-x_{\max , o}+M_{b i g} t_{t, o, 2} \\
y_{t, c} \leq y_{\min , o}+M_{b i g} t_{t, o, 3} \\
-y_{t, c} \leq-y_{\max , o}+M_{b i g} t_{t, o, 4} \\
z_{t, c} \leq z_{\min , o}+M_{b i g} t_{t, o, 5} \\
-z_{t, c} \leq-z_{\max , o}+M_{b i g} t_{t, o, 6} \\
\sum_{k=1}^{6} t_{t, o, k} \leq 5
\end{array}
$$

where $T$ is the number of time steps considered for the UAV to move through the finite horizon and $O$ is the number of the obstacles indicated by the ADS-B as the intruder aircraft. Furthermore, $\forall k \in[1,, 6], t_{i, c, k}$ is a binary variable and the last constraint in equation 33 ensures that at least one constraint is active at every time step.

\subsection{Defining Waypoints to Visit in Fi- nite Horizon}

The waypoints that the UAVs need to visit can be added to the MILP formulation in the form of the following constraints:

$$
\begin{array}{r}
\forall t \in[0, \ldots, T], \forall w_{c} \in\left[1, \ldots, W_{c}\right] \\
x_{c, t}-x_{w_{c}} \leq M_{b i g}\left(1-b_{t, w_{c}}\right) \\
x_{c, t}-x_{w_{c}} \geq-M_{b i g}\left(1-b_{t, w_{c}}\right) \\
y_{c, t}-y_{w_{c}} \leq M_{b i g}\left(1-b_{t, w_{c}}\right) \\
y_{c, t}-y_{w_{c}} \geq-M_{b i g}\left(1-b_{t, w_{c}}\right) \\
z_{c, t}-z_{w_{c}} \leq M_{b i g}\left(1-b_{t, w_{c}}\right) \\
z_{c, t}-z_{w_{c}} \geq-M_{b i g}\left(1-b_{t, w_{c}}\right) \\
\sum_{t=1}^{T} b_{t, w_{c}}=1
\end{array}
$$

The above constraints, where $\left(x_{c, t}, y_{c, t}, z_{c, t}\right)$ is the position of the UAV $c$ and $\left(x_{w_{c}}, y_{w_{c}}, z_{w_{c}}\right)$ is the position of the predefined waypoint inside the finite horizon, $b_{t, w_{c}}$ is a binary variable which indicates whether a waypoint $w_{c}$ is visited at time step t or not and the last constraint enforces the UAV to visit the waypoint just once. Often, the above constraints are relaxed by adding a value $\delta$ in order to mark a particular point as visited when the UAV comes within $\delta$ neighborhood of the waypoint.

\subsection{Technique for Avoiding Corners of Obstacles}

There are two possible corner scenarios mentioned in literatures. In first one, the obstacles can be modeled more accurately by adding more constraints [16] which results in more computations. The second approach focuses on adding safety margin around the obstacles [2]. Safety Margin for flight around the obstacles for the purpose of avoiding the corner can be found as mentioned in 35 :

$$
\operatorname{SafetyMargin}(S M) \geq \frac{v_{\max } T_{d}}{2} \cdot \sin (\gamma)
$$

where $v_{\max }$ is the maximum velocity of $\mathrm{UAV}, T_{d}$ is the sample time of discretization and $\gamma$ is the angle between the boundary and the path between the waypoints. 


\subsection{Cost Function in MILP}

Here, we use the standard cost function proposed by [35] for minimizing time:

$$
\begin{array}{r}
J_{1 . c}=\sum_{t=0}^{T}\left[-x_{c, t}^{\prime}\left(x_{w_{c}}-x_{c, 0}+\left|x_{c, t}-x_{w_{c}}\right|\right)\right. \\
\left.-y_{c, t}^{\prime}\left(y_{w_{c}}-y_{c, 0}+\left|y_{c, t}-y_{w_{c}}\right|\right)\right]
\end{array}
$$

Here, $x_{c, 0}$ and $y_{c, 0}$ indicates the starting point in finite horizon, $x_{w_{c}}$ and $y_{w_{c}}$ the next waypoint (upon existence in finite horizon).

The cost function for UAV $c$ for minimizing fuel/energy consumption can be added to the overall cost function. Consider $f_{x, t, c}, f_{y, t, c}$ and $f_{z, t, c}$ are the components of the forces applied on the UAV $c$ at time $t$ (assuming the energy consumption is directly proportional to magnitude of forces applied on the UAV). Since absolute value (representing the magnitude) in the cost function causes non-linearity, it needs to be transformed as mentioned in [41, 44, 43]. The resulting equations representing the cost function and the constraints are given by:

$$
\begin{aligned}
J_{2, c} & =\sum_{t=1}^{T} w_{1, t, c}+w_{2, t, c}+w_{3, t, c} \\
f_{x, t, c} & \leq w_{1, t, c} \\
-f_{x, t, c} & \leq w_{1, t, c} \\
f_{y, t, c} & \leq w_{2, t, c} \\
-f_{y, t, c} & \leq w_{2, t, c} \\
f_{z, t, c} & \leq w_{3, t, c} \\
-f_{z, t, c} & \leq w_{3, t, c}
\end{aligned}
$$

where $w_{1, t, c}, w_{2, t, c}$ and $w_{3, t, c}$ are slack variables. And the total cost function, that comprises both time and energy, is represented by Equation 38 .

$$
\begin{array}{r}
\forall c \in[1, \ldots, C] \\
J_{\text {total }}=\sum_{c=1}^{C} J_{1, c}+\sum_{c=1}^{C} J_{2, c}
\end{array}
$$

\subsection{MILP Solution}

For solving Mixed Integer Programming (MIP), two methods have been proposed for obtaining exact so- lutions in the literature. First one is the pure rational approach [3] and the second is the safe floating-point (FP) approach $[14,30]$. Both of these methods, utilize branch-and-bound technique originally used to solve Linear Programming (LP) problems. In pure rational approach, by performing all arithmetic operations over the rationals and applying an exact LP solver, correctness is achieved. On the other hand, safe Floating-Point (FP) based method for solving MIP [14] has been extensively used for reducing the time of computation [15]. The pure rational approach for solving in MIP is always applicable but introduces a large overhead in running time while the safe-FP approach is more efficient but of limited applicability for complex problems.

This paper proposes an algorithm that is based on two parts: the main and the slave program. Implementation of the rational approach is done in main process. During the slave process, the faster safe-FP approach is applied. To achieve reasonable running time whenever possible the expensive rational computation of the main process is skipped and certain decisions from the faster safe-FP process is substituted. The overview of the exact algorithm for solving this problem is provided in Algorithm 1.

In particular, dual bound computations need exact linear programming (LP) solutions via the rational approach, and since this approach is computationally extensive, it is avoided. The substitution for dual bounds is calculated using the safe-FP approach. Consider $P$ as the set of all possible answers and $c$ as objective value. Furthermore $c^{*}$ is the optimum objective value. $\widetilde{P}$ is the complementary of $P$ and $k$ and $i$ are nodes in the acceptable area. The slave process is implemented on a MIP instance with FP-representable data. If the input data are already FP-representable, both processes solve the same MIP instance, i.e., $\widetilde{P}:=P$ and $\widetilde{c}:=c$ in slave process. An approximation of the MIP with $P \approx \widetilde{P}, c \approx \widetilde{c}$ or a relaxation with $P \subseteq \widetilde{P}$ and $c \subseteq \widetilde{c}$ is constructed, which are called FP-approximation and FP-relaxation, respectively. The program applies safe-FP approach for obtaining the necessary exact LP solutions.

On the implementation side, the algorithm maintains only one single branch-and-bound tree. At the root node of this common tree, the LP relaxations of 
Consider $P$ as the set of possible answers according to the constraints Consider the FP-problem, provide $\widetilde{P}$ and store $\max \left\{\widetilde{c}^{T} x: x \in \widetilde{P}\right\}$

Put $\varpi=(P, \widetilde{P}), L=-\infty, c^{M I P}:=-\infty$,

Choose $k$ randomly in the area

while the Solution $c$ is not acceptable do if $\varpi=0$ then stop and return $c^{M I P}$ and solution else

choose $\left(P_{k}, \widetilde{P}_{k}\right) \in \varpi$ solve dual bound LP-relaxation of $\max \left\{\widetilde{c}^{T} x: x \in \widehat{L P}_{k}\right\}$

Check the Bounding for acceptability of the solution, check the primal bound, whether $c^{M I P}:=c^{*}$

end

For branching use these formulas

(a) Split $P_{k}$ in $Q_{1}:=\left\{P_{k} \bigcap x: x_{i} \leq\left\lfloor x_{i}^{*}\right\rfloor\right\}$, $Q_{2}:=\left\{P_{k} \bigcap x: x_{i} \geq\left\lceil x_{i}^{*}\right\rceil\right\}$.

(b) Split $\widetilde{P}_{k}$ in $\widetilde{Q}_{1}:=\left\{\widetilde{P}_{k} \bigcap x: x_{i} \leq\left\lfloor x_{i}^{*}\right\rfloor\right\}$, $\widetilde{Q}_{2}:=\left\{\widetilde{P}_{k} \bigcap x: x_{i} \geq\left\lceil x_{i}^{*}\right\rceil\right\} ;$ set $\varpi:=\varpi \cup\left\{\left(\left(Q_{1}, \widetilde{Q}_{1}\right),\left(Q_{2}, \widetilde{Q}_{2}\right)\right)\right\}$; end

Print the solution

Algorithm 1: Algorithm for fast solution of MILP both processes including $\max \left\{\widetilde{c}^{T} x: x \in \widetilde{L P}_{k}\right\}$ and $\max \left\{\widetilde{c}^{T} x: x \in \widetilde{P}\right\}$ are stored. In addition, for each node, it is known which branching constraint was introduced to create both sub-problems. Algorithm 1 identifies primal solutions by checking LP solutions for integrality.

\subsection{Path Smoother}

The path obtained by the MILP based planning algorithm is in the form of waypoints. The path joining these waypoints (as shown by dotted lines in Figure 2 ) is piece-wise linear and hard to be tracked by a specific UAV $c$ with dynamic and kinematic constraints. Hence, it is necessary to make the path smoother for the points generated from the path planning algorithm in order to be suitable for UAVs. A rotorcraft UAV can fly in linear segments at low speed with the capability to stop and hover at each waypoint. However, at higher speeds, UAV is not capable of negotiating turns at smaller radii, which imposes the demand for a planner that accommodates for nonholonomic constraints [52]. Essentially the aim is to generate a path which satisfies the maximum curvature constraint of the UAV [6].

For the solution of this problem $C^{1}$ Continuous Cubic Bezier Curve (C1CBC) method with some modifications is used. Let the degree $n$ Bezier curve with $n+1$ control points $\left(P_{0}, P_{1}, \ldots, P_{n}\right)$ be defined as $[6]$ :

$$
P(S)=\sum_{i=0}^{n} P_{i} B_{n, i}(s)
$$

where $B_{n, i}(s)$ are named Berstein polynomials calculated by:

$$
B_{n, i}(s)=\left(\begin{array}{c}
n \\
i
\end{array}\right) s^{i}(1-s)^{n-i}
$$

Lower degree of Bezier curves is preferable, due to the cost of time computation. Four control points $\left(P_{0}, P_{1}, P_{2}, P_{3}\right)$ are needed to make a cubic Bezier curve, where $P_{0}$ and $P_{3}$ are the curve end points. For making the curves continuous, it is necessary for 
the first derivative of the two curves at the junction points to be the same. The derivative represented by:

$$
\frac{d}{d s} P(s)=\sum_{i=0}^{n-1} n\left(P_{i+1}-P_{i}\right) B_{n-1, i}(s)
$$

Due to collinear and equally spaced curves, the first and second control points $P_{0}$ and $P_{1}$ must be located between $\operatorname{Pos}_{U A S, t}=\left(x_{t}, y_{t}\right)$ and $\operatorname{Pos}_{U A S, t+1}=$ $\left(x_{t+1}, y_{t+1}\right)$ and the last two control points $P_{2}$ and $P_{3}$ must be placed between $\operatorname{Pos}_{U A S, t+1}=\left(x_{t+1}, y_{t+1}\right)$ and $\operatorname{Pos}_{U A S, t+2}=\left(x_{t+2}, y_{t+2}\right)$. The locations of four control points are indicated as following:

$$
\begin{aligned}
P_{0} & =\operatorname{Pos}_{U A S, t+1}+d_{1} \cdot u_{1} \\
P_{1} & =\operatorname{Pos}_{U A S, t+1}+\frac{d_{1} \cdot u_{1}}{2} \\
P_{2} & =\operatorname{Pos}_{U A S, t+1}-\frac{d_{2} \cdot u_{2}}{2} \\
P_{3} & =\operatorname{Pos}_{U A S, t+1}-d_{2} \cdot u_{2}
\end{aligned}
$$

In which $u_{1}$ is a unit vector between $\operatorname{Pos}_{U A S, t+1}$ and $\operatorname{Pos}_{U A S, t}$ and $u_{2}$ is between $\operatorname{Pos}_{U A S, t+1}$ and $\operatorname{Pos}_{U A S, t+2} . \quad d_{1}$ is the length between $\operatorname{Pos}_{U A S, t+1}$ and $P_{0}$ and $d_{2}$ is that between $P_{0} s_{U A S, t+1}$ and $P_{3}$. Figure 2 represents the control points and schematic of the path smoother strategy.

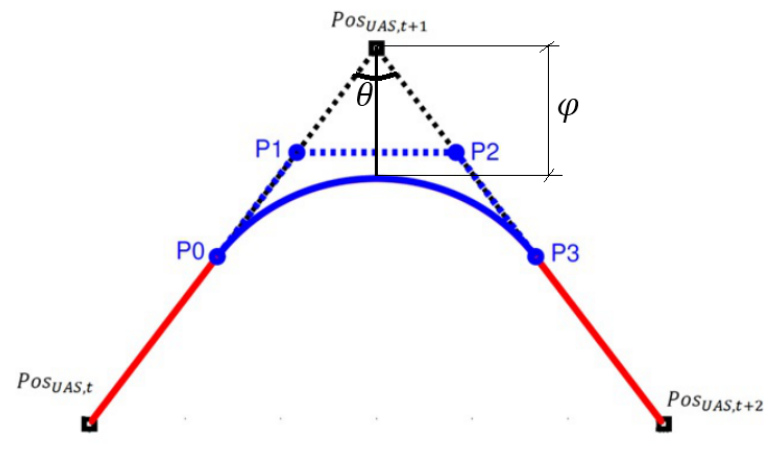

Figure 2: Control points and schematic of the path smoother strategy.

This point generation goes on till the UAV meets its requirement for turning. The piece-wise linear form of the path is shown in Figure 3.

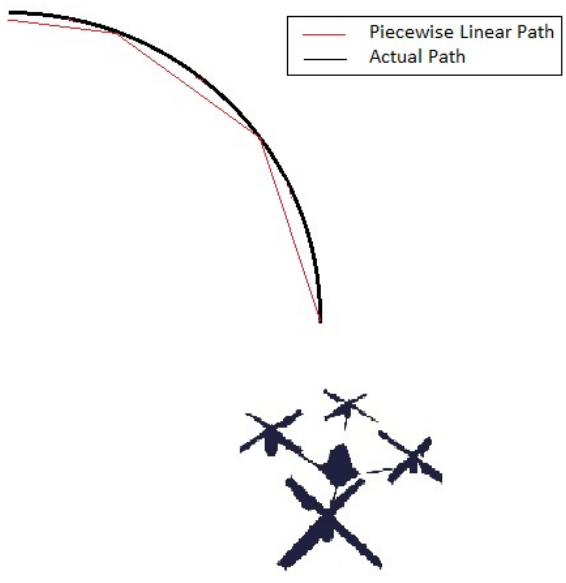

Figure 3: Schematic diagram showing the piecewise linear path joining the waypoints and the path obtained by the path smoother.

The angle $\theta$ which represents the angle between the maximum discretized points can be defined by:

$$
\theta=\cos ^{-1}\left(\frac{u_{1} \cdot u_{2}}{\left|u_{1}\right|\left|u_{2}\right|}\right)
$$

The velocity at entire piece-wise linear path generated by this method remains constant and equal to the velocity of UAV at $\operatorname{Pos}_{U A S, t}$.

The maximum deviation from the path exerted on $\operatorname{Pos}_{U A S, t+1}$ and in figure 2 is shown by $\phi$. This depends on the angle the UAV is trying to turn. The maximum deviation is:

$$
\phi=\frac{\rho_{1} \tan \theta(1-\sin \theta)}{\sin \theta}
$$

By this method, there is a chance that in the positions of $P_{0}$ and $P_{3}$, shown in figure 2 , the constraint of minimum angle is not satisfied. For overcoming this problem the produced curvature needs to be refined till this constraint is satisfied. 


\section{Results and Flight Simula- tions}

This section presents results obtained from extensive numerical simulations carried out with varying number of UAVs with varying obstacle scenarios. Simulations showing linear, trapezoidal and triangular formations have been carried out. These simulations make the following assumptions:

1. The pre-defined path is set to be a straight line.

2. The discretized time is considered to be one second.

3. There is a safety margin around each fixed obstacle and IA which is assumed to be ten meters.

4. The maximum and minimum speeds are set to be $35 \mathrm{~m} / \mathrm{s}$ and $5 \mathrm{~m} / \mathrm{s}$, respectively.

In this paper the simulations have been done on a computer with the following specifications:

1. Operating System: Windows 7 enterprise 64-bit

2. Program language: MATLAB R2014a

3. MILP solver: Gurobi Optimizer 4.5

4. Simulation environment: YALMIP

5. Processor: Intel(R) Core(TM)i7 -2500 CPU @ $3.30 \mathrm{GHz}$

6. (2 CPUs)

7. Memory: 8.00 GB RAM

The parameters used for simulation are as mentioned here:

- $M_{b i g}=1000$

- $Q_{4}=Q_{1}=10$

- $r=1.05$

- $Q_{2}=16$

- $Q_{3}=5$
- $S F=5$

- $r_{\text {threat }}=75$ meter

- $\gamma=45$

\subsection{Formation Constraints}

Two different scenarios have been considered for three kinds of formations, e.g. linear, trapezoid and triangular. In the first scenario, the formation constraint would be relaxed for a limited time when the UAV team faces obstacles in the path and then would revert to the same formation. So the algorithm is implemented in the way that if one of the UAVs senses the collision danger, then all the UAVs would be released from the constraints due to formation and predefined path. The UAVs get back to the goal positions once the collision danger is passed. The second scenario considered in the paper is when the formation constraint is applied at all times and the UAVs with the same formation are trying to change their path in presence of fixed and moving obstacles. For each scenario, the simulation has been carried out for the mentioned three different formation shapes. The time of computation using the proposed method of finite horizon has been compared with the method that does not utilizie finite horizon and carries out the computation for the entire air-space.

\subsubsection{Linear Formation}

The scenario has been designed as the UAVs start from a linear formation. The constraint added to the problem is:

$$
\begin{array}{r}
\forall c \in[2, \ldots, C], \forall t[0, \ldots, T] \\
\left|X_{c, t}-X_{c-1, t}\right|=P \\
\left|Y_{c, t}-Y_{c-1, t}\right|=0
\end{array}
$$

where $C$ represents the number of UAVs cooperating in the formation and $T$ is the time taken for UAVs to reach the goal. Furthermore, $P$ is a constant which represents the characteristics of linear formation of the UAVs. In Figure 4, the UAVs can break the formation and reach the goal (Scenario 1) and in Figure 5 the UAVs change their path whilst they are moving 
in a constrained linear formation (Scenario 2).

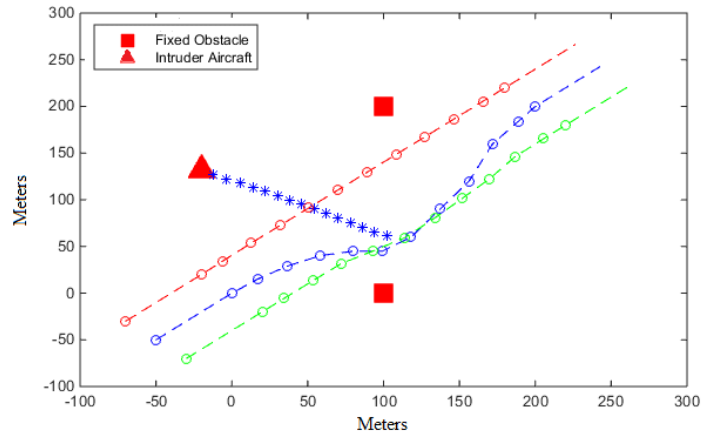

(a) Formation Using the Fast Dynamic MILP Method (Scenario 1)

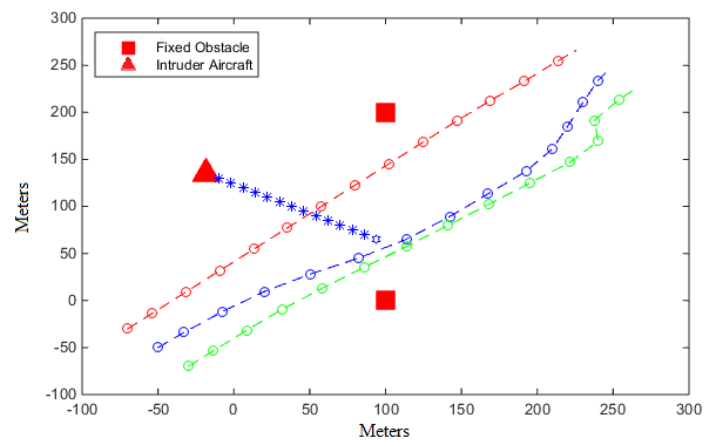

(b) Formation Without Using the Fast Dynamic MILP Method (Scenario 1)

Figure 4: Linear formation in presence of fixed and moving obstacles (Scenario 1)

As shown in figure 4 , the UAV in the center senses the intrusion and tries to change its path. Simultaneously, the UAVs are avoiding colliding with each other. Therefore, the UAV on the right hand side also changes its own path to maintain separation from the UAV in the center. It may be noted that the UAVs do not collide with each other.

The comparison between the time of computations has been represented in Figure 6 .

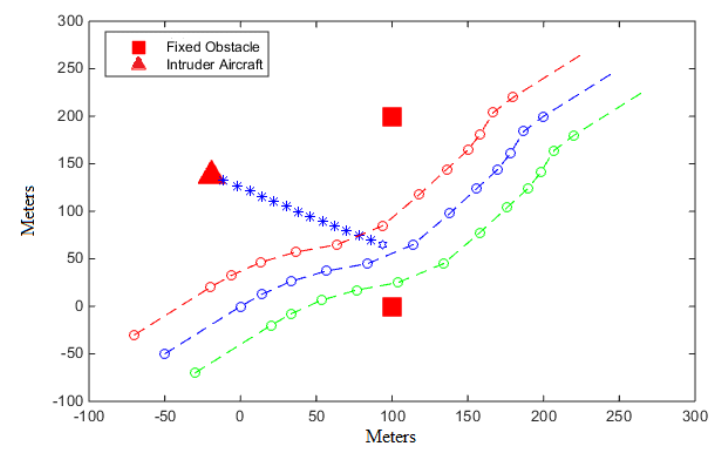

(a) Formation Using the Fast Dynamic MILP Method (Scenario 2)

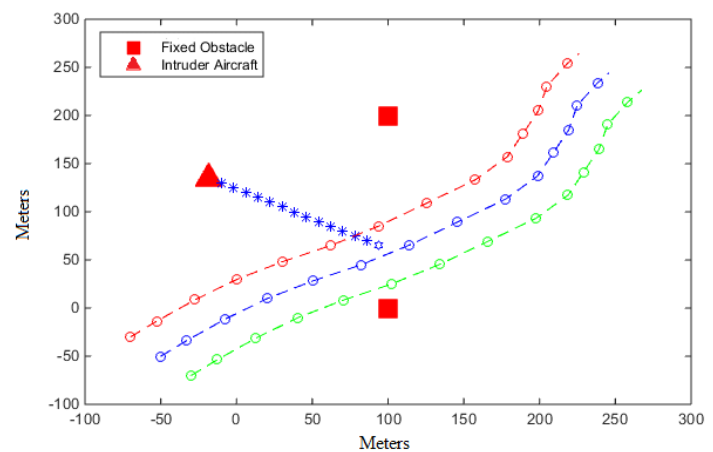

(b) Formation Without Using the Fast Dynamic MILP Method (Scenario 2)

Figure 5: Constrained linear formation in presence of fixed and moving obstacles (Scenario 2)

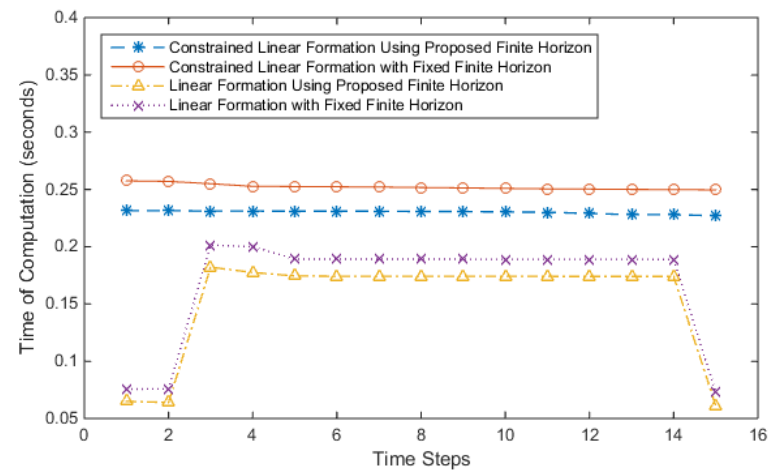

Figure 6: Comparison between the time of computation for the different methods 


\subsubsection{Triangular Formation}

In this formation the UAVs are cooperating in a triangular formation and try to find the optimal trajectory in presence of fixed obstacles and IA. The constraint for this formation is:

$$
\begin{array}{r}
\forall c \in[2, \ldots, C], \forall t[0, \ldots, T] \\
\left|X_{c, t}-X_{c-1, t}\right|=P \\
\left|Y_{c, t}-Y_{c-1, t}\right|=P
\end{array}
$$

The results for the formation without the constraints (Scenario 1) of the formation are presented in Figure 7. Also, the constrained formations (Scenario 2) have been shown in Figure 8.

The comparison between times of computation has been presented in Figure 9.

Similar to linear formation (Scenario 1), the UAV on the left hand side does not sense any collision in its path planning whilst the UAV in the center senses that there might be a collision and modifies its path. However, in this case, the UAV on the right does not need to modify its path.

\subsubsection{Trapezoid Formation}

In this scenarios the UAVs are avoiding the fixed obstacles and IAs while cooperating in a trapezoid formation. The constraint for the trapezoid formation is as:

$$
\begin{array}{r}
\forall c \in[2, \ldots, C], \forall t[0, \ldots, T] \\
\left|X_{c, t}-X_{c-1, t}\right|=P
\end{array}
$$

Figure 10 presents the trajectories, found in absence of the formation constraint (Scenario 1) and Figure 11 shows the trajectories of UAVs in presence of the formation constraint (Scenario 2).

As shown in figure 10, the algorithm is capable of solving complex scenarios in a time-efficient manner.

And the time of computation for each method has been presented in Figure 12 .

It may be seen that the computational time requirement initially is small for fast dynamic MILP method, because finite horizon is not formed initially (it only forms when an obstacle or IA is detected). Upon

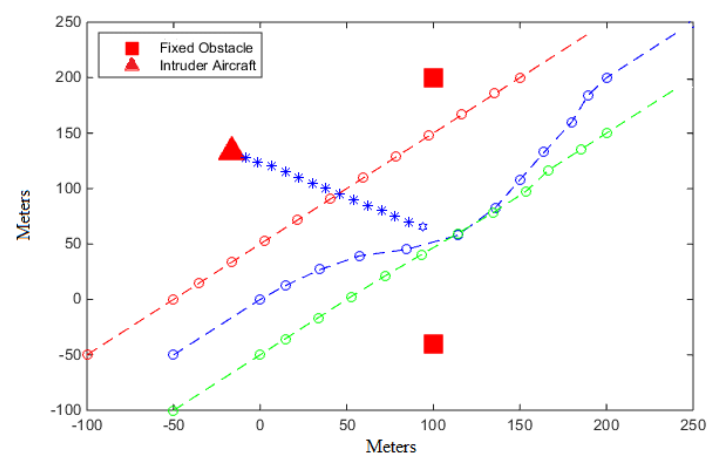

(a) Formation Using the Fast Dynamic MILP Method (Scenario 1)

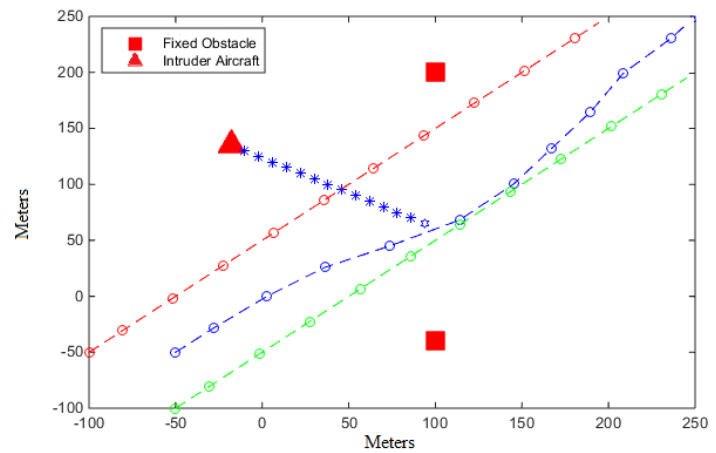

(b) Formation Without Using the Fast Dynamic MILP Method (Scenario 1)

Figure 7: Triangular formation in presence of fixed and moving obstacles (Scenario 1) 


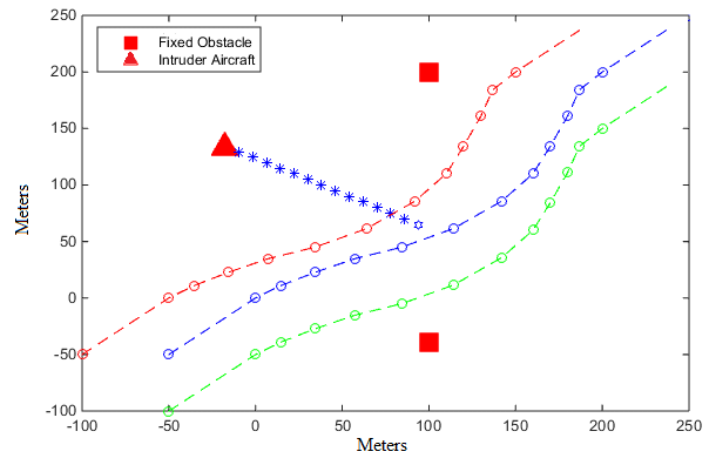

(a) Formation Using the Fast Dynamic MILP Method (Scenario 2)

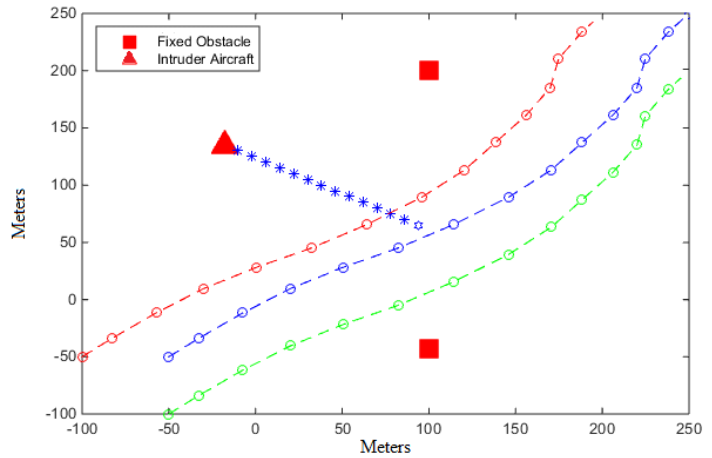

(b) Formation Without Using the Fast Dynamic MILP Method (Scenario 2)

Figure 8: Constrained Triangular formation in presence of fixed and moving obstacles (Scenario 2)

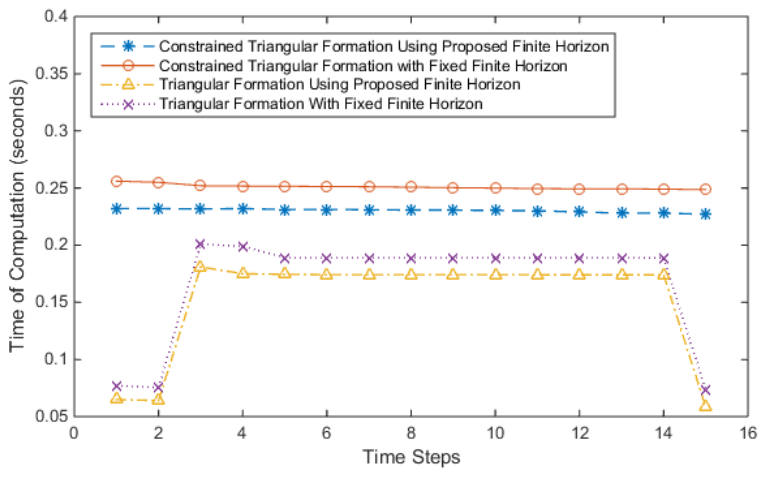

Figure 9: Time of computation for triangular formation of UAVs for the different method

detection of obstacle, the dynamic finite horizon is formed and time requirement bumps up. Finally, the time requirement goes down again when no obstacles are detected towards the end of the flight. For Method 1, where a fixed finite horizon of size equal to entire air-space is present all time, computational time requirement remains almost the same throughout the flight.

It can be seen from the results that the computational time requirement for Method 2 (dynamic finite horizon) is much less than the computational time requirement for Method 1 (fixed finite horizon). This demonstrates that the local strategy for path planning with dynamic finite horizon is as optimal as fixed finite horizon but requires lesser computational time. This is significant because the method needs to be applied on-board for path planning with limited computational resources and real-time requirement.

\section{Conclusion}

This paper proposes an approach that utilizes the formulation of fast-dynamic MILP and path smoother to solve the path planning and collision avoidance for a fleet of UAVs flying in formation. The problem solved in this paper is relevant to problem of sense and avoid where pre-defined trajectory of a UAV would need to be modified to prevent collisions from other aircraft. The paper assumes the knowl- 


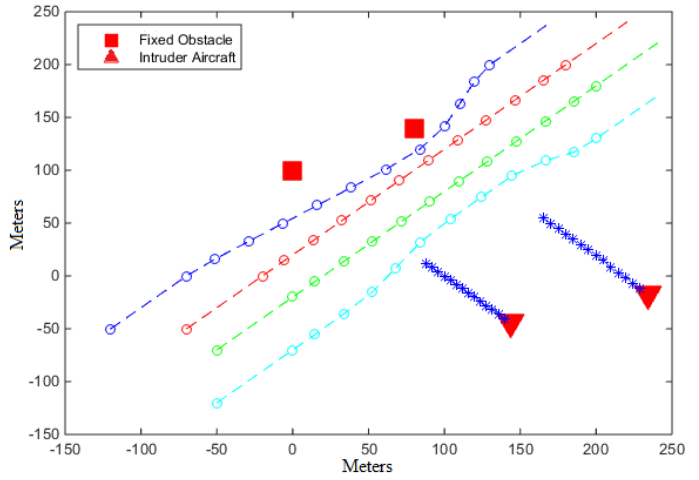

(a) Formation Using the Fast Dynamic MILP Method (Scenario 1)

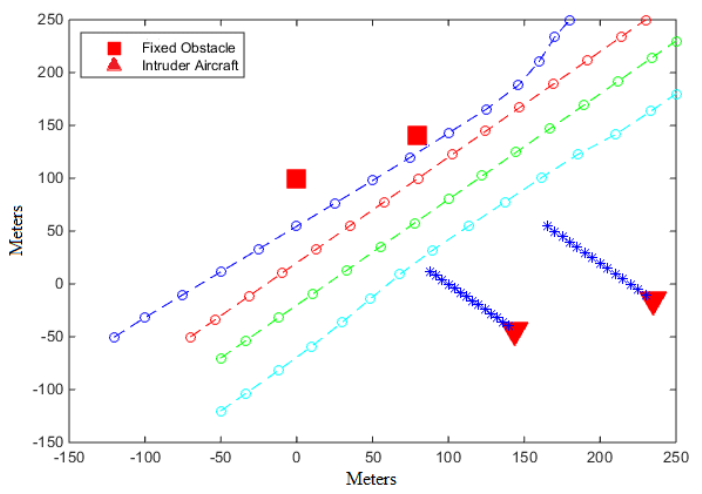

(b) Formation Without Using the Fast Dynamic MILP Method (Scenario 1)

Figure 10: Trapezoid formation in presence of fixed and moving obstacles (Scenario 1)

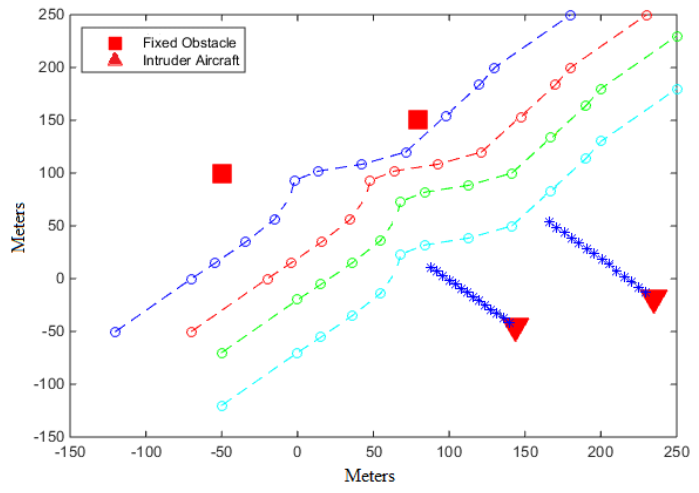

(a) Formation Using the Fast Dynamic MILP Method (Scenario 2)

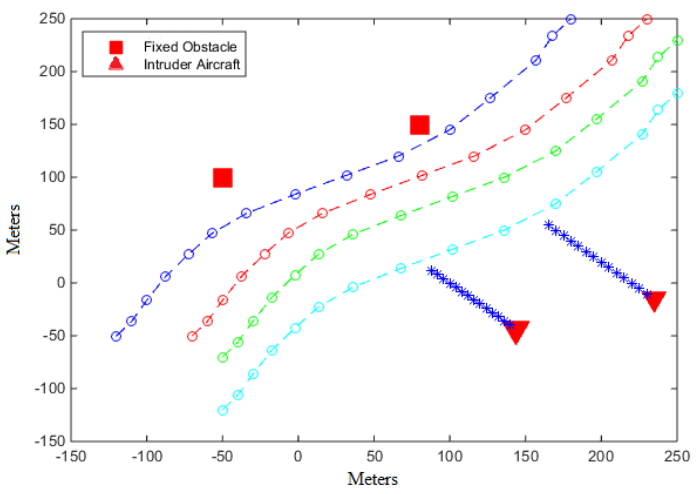

(b) Formation Without Using the Fast Dynamic MILP Method (Scenario 2)

Figure 11: Constrained trapezoid formation in presence of fixed and moving obstacles (Scenario 2) 


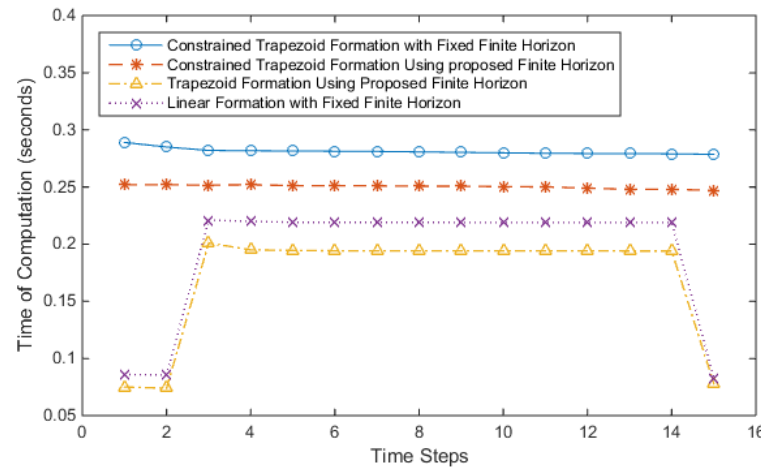

Figure 12: Time of computation for trapezoid formation of UAVs for the different method

edge of location of obstacles and other aircraft, which in real world scenario, would be obtained from ADS$\mathrm{B}$ or onboard sensors. Based on this data and estimation of other aircrafts velocity/acceleration, it is determined if the aircraft would be a threat for collision. Based on this, the path of the UAVs is improvised by defining a finite horizon. The path planning problem is then formulated as fast-dynamic MILP with obstacles (both stationary and dynamic ones) incorporated into the optimization problems as constraints. Two different scenarios, i.e., fixed formation (formation does not change) and flexible formation (formation shape changes) have been considered in this paper. Similarly, the waypoints are incorporated as constraints in the problem formulation. The cost function used for the optimization comprises of two components: total time of flight and energy/fuel consumption. The numerical results obtained from several scenarios demonstrate the ability of the program to carry out the path planning for a variety of flight missions.

\section{References}

[1] Mehdi Alighanbari, Yoshiaki Kuwata, and Jonathan P How. Coordination and control of multiple UAVs with timing constraints and loitering. In American Control Conference, 2003.
Proceedings of the 2003, volume 6, pages 53115316. IEEE, 2003.

[2] PE An, CJ Harris, R Tribe, and N Clarke. Aspects of neural networks in intelligent collision avoidance systems for prometheus. 1993.

[3] David L Applegate, William Cook, Sanjeeb Dash, and Daniel G Espinoza. Exact solutions to linear programming problems. Operations Research Letters, 35(6):693-699, 2007.

[4] R Asep, AK Achaibou, and F Mora-Camino. Automatic collision avoidance based on supervised predictive controllers. Control Engineering Practice, 4(8):1169-1175, 1996.

[5] Tucker Balch and Ronald C Arkin. Behaviorbased formation control for multirobot teams. Robotics and Automation, IEEE Transactions on, 14(6):926-939, 1998.

[6] John C Beatty and Brian A Barsky. An introduction to splines for use in computer graphics and geometric modeling. Morgan Kaufmann, 1995.

[7] John S Bellingham, Michael Tillerson, Mehdi Alighanbari, and Jonathan P How. Cooperative path planning for multiple UAVs in dynamic and uncertain environments. In Decision and Control, 2002, Proceedings of the 41st IEEE Conference on, volume 3, pages 2816-2822. IEEE, 2002.

[8] William Blake and Dieter Multhopp. Design, performance and modeling considerations for close formation flight. CLJ, 150:2, 1998.

[9] Louis E Buzogany, M Pachter, and JJ dAzzo. Automated control of aircraft in formation flight. In Proc. AIAA Guidance, Navigation, and Control Conference, pages 1349-1370, 1993.

[10] Atif Chaudhry, Kathy Misovec, and Raffaello D'Andrea. Low observability path planning for an Unmanned Air Vehicle using mixed integer linear programming. In Decision and Control, 2004. CDC. 43rd IEEE Conference on, volume 4, pages 3823-3829. IEEE, 2004. 
[11] Luciano E Chiang. 3-d CNC trajectory interpolation using bresenham's algorithm. In Industrial Electronics, 1994. Symposium Proceedings, ISIE'94., 1994 IEEE International Symposium on, pages 264-268. IEEE, 1994.

[12] David F Chichka and Jason L Speyer. Solarpowered, formation-enhanced aerial vehicle systems for sustained endurance. In American Control Conference, 1998. Proceedings of the 1998, volume 2, pages 684-688. IEEE, 1998.

[13] John C Clements. The optimal control of collision avoidance trajectories in air traffic management. Transportation Research Part B: Methodological, 33(4):265-280, 1999.

[14] William Cook, Sanjeeb Dash, Ricardo Fukasawa, and Marcos Goycoolea. Numerically safe gomory mixed-integer cuts. INFORMS Journal on Computing, 21(4):641-649, 2009.

[15] William Cook, Thorsten Koch, Daniel E Steffy, and Kati Wolter. An exact rational mixedinteger programming solver. In Integer Programming and Combinatoral Optimization, pages 104-116. Springer, 2011.

[16] Matthew G Earl and Raffaello D'Andrea. Iterative MILP methods for vehicle-control problems. Robotics, IEEE Transactions on, 21(6):11581167, 2005.

[17] Nadeem Faiz, Sunil K Agrawal, and Richard M Murray. Trajectory planning of differentially flat systems with dynamics and inequalities. Journal of Guidance, Control, and Dynamics, 24(2):219 $227,2001$.

[18] John T Feddema, Chris Lewis, David Schoenwald, et al. Decentralized control of cooperative robotic vehicles: theory and application. Robotics and Automation, IEEE Transactions on, 18(5):852-864, 2002.

[19] Erik J Forsmo, Esten Grøtli, Thor Fossen, Tor Arne Johansen, et al. Optimal search mission with Unmanned Aerial Vehicles using mixed integer linear programming. In Unmanned Aircraft Systems (ICUAS), 2013 International Conference on, pages 253-259. IEEE, 2013.

[20] Thor I Fossen. Handbook of marine craft hydrodynamics and motion control. John Wiley \& Sons, 2011.

[21] Leonid B Freidovich and Hassan K Khalil. Lyapunov-based switching control of nonlinear systems using high-gain observers. Automatica, 43(1):150-157, 2007.

[22] Esten Ingar Grøtli and Tor Arne Johansen. Path planning for UAVs under communication constraints using SPLAT! and MILP. Journal of Intelligent \& Robotic Systems, 65(1-4):265-282, 2012.

[23] Gautier Hattenberger, Rachid Alami, and Simon Lacroix. Planning and control for Unmanned Air Vehicle formation flight. In Intelligent Robots and Systems, 2006 IEEE/RSJ International Conference on, pages 5931-5936. IEEE, 2006.

[24] Hassan K Khalil and JW Grizzle. Nonlinear systems, volume 3. Prentice hall New Jersey, 1996.

[25] Feng-Li Lian and Richard Murray. Real-time trajectory generation for the cooperative path planning of multi-vehicle systems. In Decision and Control, 2002, Proceedings of the 41st IEEE Conference on, volume 4, pages 3766 3769. IEEE, 2002.

[26] Johan Löfberg. YALMIP: A toolbox for modeling and optimization in MATLAB. In Computer Aided Control Systems Design, 2004 IEEE International Symposium on, pages 284-289. IEEE, 2004

[27] Akira Miura, Hiroyuki Morikawa, and Moriyuki Mizumachi. Aircraft collision avoidance with potential gradientground-based avoidance for horizontal maneuvers. Electronics and Communications in Japan (Part III: Fundamental Electronic Science), 78(10):104-114, 1995. 
[28] KS Mostov and AA Soloviev. Fuzzy adaptive stabilization of higher order kalman filters in application to precision kinematic GPS. In Proceedings of the 9th International Technical Meeting of the Satellite Division of The Institute of Navigation (ION GPS 1996), pages 1451-1456, 1996.

[29] Alireza Nemati and Manish Kumar. Control of microcoaxial helicopter based on a reducedorder observer. Journal of Aerospace Engineering, page 04015074, 2015.

[30] Arnold Neumaier and Oleg Shcherbina. Safe bounds in linear and mixed-integer linear programming. Mathematical Programming, 99(2):283-296, 2004.

[31] Francesco Nex and Fabio Remondino. Uav for 3d mapping applications: a review. Applied Geomatics, 6(1):1-15, 2014.

[32] Bruce D Nordwall. Road map leads FAA to freeflight. AVIATION WEEK \& SPACE TECHNOLOGY, 143(19):34-34, 1995.

[33] Lynne E Parker. Current state of the art in distributed autonomous mobile robotics. In Distributed Autonomous Robotic Systems 4, pages $3-12$. Springer, 2000.

[34] Tekla S Perry. In search of the future of air traffic control. Spectrum, IEEE, 34(8):18-35, 1997.

[35] Mohammadreza Radmanesh, Manish Kumar, Alireza Nemati, and Mohammad Sarim. Dynamic optimal UAV trajectory planning in the national airspace system via mixed integer linear programming. Proceedings of the Institution of Mechanical Engineers, Part G: Journal of Aerospace Engineering, page 0954410015609361, 2015.

[36] Mohammadreza Radmanesh, Alireza Nemati, Mohammad Sarim, and Manish Kumar. Flight formation of quad-copters in presence of dynamic obstacles using mixed integer linear programming. In Dynamic Systems and Control Conference, Proceedings of the DSCC2015.
American Society of Mechanical Engineering, 2015.

[37] Mohammadreza Radmanesh, Omid Nematollahi, Mahdi Nili-Ahmadabadi, and Mostafa Hassanalian. A novel strategy for designing and manufacturing a fixed wing MAV for the purpose of increasing maneuverability and stability in longitudinal axis. Journal of Applied Fluid Mechanics, 7(3):435-446, 2014.

[38] Robin L Raffard, Claire J Tomlin, and Stephen P Boyd. Distributed optimization for cooperative agents: Application to formation flight. In Decision and Control, 2004. CDC. 43rd IEEE Conference on, volume 3, pages 24532459. IEEE, 2004.

[39] Ingeborgl Ray. ADS integration into the flight management computer. In FAA, The First Annual International Satellite Surveillance and Communication Symposium p 277-280(SEE N 91-32093 24-04), 1991.

[40] D Reichardt and J Shick. Collision avoidance in dynamic environments applied to autonomous vehicle guidance on the motorway. In Intelligent Vehicles' 94 Symposium, Proceedings of the, pages 74-78. IEEE, 1994.

[41] Christian Reinl and Oskar Von Stryk. Optimal control of multi-vehicle-systems under communication constraints using mixed-integer linear programming. In Proceedings of the 1st international conference on Robot communication and coordination, page 3. IEEE Press, 2007.

[42] Arthur Richards, John Bellingham, Michael Tillerson, and Jonathan How. Coordination and control of multiple UAVs. In AIAA guidance, navigation, and control conference, Monterey, CA, 2002.

[43] Arthur Richards and Jonathan P How. Aircraft trajectory planning with collision avoidance using mixed integer linear programming. In American Control Conference, 2002. Proceedings of the 2002, volume 3, pages 1936-1941. IEEE, 2002. 
[44] Arthur Richards, Tom Schouwenaars, Jonathan $\mathrm{P}$ How, and Eric Feron. Spacecraft trajectory planning with avoidance constraints using mixed-integer linear programming. Journal of Guidance, Control, and Dynamics, 25(4):755-764, 2002.

[45] Mohammad Sarim, Alireza Nemati, Manish Kumar, and Kelly Cohen. Extended kalman filter based quadrotor state estimation based on asynchronous multisensor data. In Dynamic Systems and Control Conference, Proceedings of the DSCC2015. American Society of Mechanical Engineering, 2015.

[46] Corey Schumacher and Sahjendra N Singh. Nonlinear control of multiple UAVs in close-coupled formation flight. AIAA paper, 4373:2000, 2000.

[47] Claire J Tomlin, John Lygeros, and S Shankar Sastry. A game theoretic approach to controller design for hybrid systems. Proceedings of the IEEE, 88(7):949-970, 2000.

[48] Berthold Ulmer. Vita ii-active collision avoidance in real traffic. In Intelligent Vehicles' 94 Symposium, Proceedings of the, pages 1-6. IEEE, 1994.

[49] Sergio Velastin, Chengping Xu, et al. Line and circle finding by the weighted mahalanobis distance transform and extended Kalman filtering. In Industrial Electronics, 1994. Symposium Proceedings, ISIE'94., 1994 IEEE International Symposium on, pages 258-263. IEEE, 1994.

[50] Oleg A Yakimenko. Direct method for rapid prototyping of near-optimal aircraft trajectories. Journal of Guidance, Control, and Dynamics, 23(5):865-875, 2000.

[51] Guang Yang and Vikram Kapila. Optimal path planning for Unmanned Air Vehicles with kinematic and tactical constraints. In Decision and Control, 2002, Proceedings of the 41st IEEE Conference on, volume 2, pages 1301- $L_{3}$ 1306. IEEE, 2002.
[52] Kwangjin Yang and Salah Sukkarieh. 3d smooth path planning for a uav in cluttered natural environments. In Intelligent Robots and Systems, 2008. IROS 2008. IEEE/RSJ International Conference on, pages 794-800. IEEE, 2008.

[53] Shannon Zelinski, T John Koo, and Shankar Sastry. Hybrid system design for formations of autonomous vehicles. In Decision and Control, 2003. Proceedings. 42nd IEEE Conference on, volume 1, pages 1-6. IEEE, 2003.

\section{List of Symbols/Abbreviations}

$\gamma \quad$ Assumed angle between the waypoints and the path

$\sigma \quad$ Parameter added to the size of obstacles for safety factor

$\tau^{b} \quad$ Control Inputs of the aircraft

$\tau_{\text {other }}$ Disturbance

$\tau_{\text {wind }}$ Forces exerted to the body caused by wind

C Total number of UAVs in formation

$D \quad$ Damping martix

$f_{\max } \quad$ Maximum force in $x y$ direction

$f_{x, t} \quad$ Force in $x$ direction exerts on the UAV

$f_{y, t} \quad$ Force in $x$ direction exerts on the UAV

$f_{z, \max }$ Maximum force in $z$ direction

$f_{z, t} \quad$ Force in $x$ direction exerts on the UAV

$g^{n} \quad$ Gravity vector in NED

$L_{1} \quad$ Length of the cube where finite horizon is generated in different scenarios

$L_{2} \quad$ Length of the cube where finite horizon is generated in different scenarios

Length of the cube where finite horizon is generated in different scenarios 
$L_{4} \quad$ Length of the cube where finite horizon is generated in different scenarios

$m \quad$ Mass of the body

$M_{b i g} \quad$ Constant number

$O \quad$ Number of IAs in finite horizon

$Q_{1} \quad$ Constant number

$Q_{2} \quad$ Constant number

$Q_{3} \quad$ Constant number

$Q_{4} \quad$ Constant number

$R \quad$ Rotation matrix

$r \quad$ Constant number

$T \quad$ Total time of flight in finite horizon

$t \quad$ Time step in finite horizon

$t_{1} \quad$ Time the UAV starts collision avoidance

$T_{d} \quad$ Sample time of discretization

$t_{G} \quad$ Time for the UAV to reach local goal

$t_{\text {collision }}$ Time predicted for the UAV till the collision

$T_{\text {total }}$ Total time of flight

$v^{b} \quad$ Velocity of object in body frame

$v_{c, t} \quad$ Velocity of UAV $c$ at time $t$

$v_{\text {max,forward }}$ Maximum velocity of UAV in $x y$ plane

$v_{\text {max }, \text {, forward }}$ Maximum velocity of IA in $x y$ plane

$v_{\text {max }, o, t o t a l}$ Maximum possible velocity of IA $o$

$v_{\max , o, z}$ Maximum velocity of IA in $z$ direction

$v_{\max , z}$ Maximum velocity of $\mathrm{UAV}$ in $z$ direction

$v_{r e l, o, t}$ Relative velocity of IA $o$ at time $t$

$v_{t, o} \quad$ Velocity of IA $o$ at time $t$

$v_{x, c, t} \quad$ Velocity of UAV $c$ in $x$ direction at time step $t$ $v_{y, c, t} \quad$ Velocity of UAV $c$ in $y$ direction at time step $t$

$v_{z, c, t} \quad$ Velocity of UAV $c$ in $z$ direction at time step $t$

$x_{c, t} \quad x$ position of UAV $c$ at time $t$

$x_{I A, t} \quad x$ position of IA at time $t$

$x_{\max , o}$ Maximum $x$ of the obstacle $o$

$x_{\max } \quad$ Maximum $x$ in finite horizon

$x_{m i n, o}$ Minimum $x$ of the obstacle $o$

$x_{\min } \quad$ Minimum $x$ in finite horizon

$x_{t_{\text {collision }}} x$ position of the prediction where collision might happens

$x_{w_{c}} \quad x$ position of the waypoint $c$

$y_{c, t} \quad y$ position of UAV $c$ at time $t$

$y_{I A, t} \quad y$ position of IA at time $t$

$y_{\max , o}$ Maximum $y$ of the obstacle $o$

$y_{\max }$ Maximum $y$ in finite horizon

$y_{\min , o}$ Minimum $y$ of the obstacle $o$

$y_{\min }$ Minimum $y$ in finite horizon

$y_{t_{\text {collision }}} y$ position of the prediction where collision might happens

$y_{w_{c}} \quad y$ position of the waypoint $c$

$z_{c, t} \quad z$ position of UAV $c$ at time $t$

$z_{I A, t} \quad z$ position of IA at time $t$

$z_{\max , o}$ Maximum $z$ of the obstacle $o$

$z_{\max }$ Maximum $z$ in finite horizon

$z_{\min , o}$ Minimum $z$ of the obstacle $o$

$z_{\min } \quad$ Minimum $z$ in finite horizon

$z_{t_{\text {collision }}} z$ position of the prediction where collision might happens

$z_{w_{c}} \quad z$ position of the waypoint $c$ 